\title{
Erratum: Chern-Simons-Higgs transitions out of topological superconducting phases [Phys. Rev. B 92, 155110 (2015)]
}

\author{
David J. Clarke and Chetan Nayak \\ (Received 24 February 2016; published 14 March 2016)
}

DOI: 10.1103/PhysRevB.93.119907

\section{INTRODUCTION}

In our continuing investigations in the same field, we have come across a significant error in our paper. While the chief results and methodology of the paper remain correct, including the majority of the worked examples, we were mistaken in the general claim that the phase transition under consideration is always between a Chern-Simons theory based on a given Lie group and the theory based on its Cartan subalgebra. The correct statement is that transitions occur between the original theory and a theory based on the stabilizer subgroup associated with the condensate. This allows for the possibility of transitions between two non-Abelian theories.

More precisely, in our paper the mass matrix for a general topological phase after superconductivity is broken is derived to be

$$
M_{\rho(\vec{g})}=\langle\rho(\vec{g}) \rho(\vec{g})\rangle-\langle\rho(\vec{g})\rangle\langle\rho(\vec{g})\rangle
$$

for the remaining gauge fluctuations. Here $\vec{g}$ is the set of generators of the original Lie algebra and $\rho$ is the representation of the original Lie group $\mathcal{G}$ carried by the condensing quasiparticle. Using the Cauchy-Schwartz inequality, one may show that the effective-mass matrix is positive semi-definite, gapping out parts of the gauge field. There is a remaining gauge symmetry if and only if $\hat{v} \cdot \rho(\vec{g})|u\rangle \propto|u\rangle$, where $|u\rangle$ is the normalized internal state vector $\left(|u\rangle=\Phi_{l}^{m} /\left|\Phi_{l}^{m}\right|\right)$ of $\Phi_{l}^{m}$. That is, there is a remaining gauge symmetry if and only if $|u\rangle$ is an eigenstate of $\hat{v} \cdot \rho(\vec{g})$ for some vector $\hat{v}$, in which case the gauge field along $\hat{v}$ remains gapless.

In our paper, the statement is made that "The number of independent vectors $\hat{v}$ satisfying this criterion is limited to be (at most) the dimension of the Cartan subalgebra $\mathcal{H} \subset \mathcal{G}$." This statement is incorrect. In fact, the number of vectors satisfying the criterion must be at least the dimension of the Cartan subalgebra. There may be more such vectors, and as a consequence the resulting ungapped subalgebra is not necessarily Abelian.

This Erratum explores the consequences of this error, and it provides a correct generalization of the transitions described in our paper to a general Lie group.

\section{GENERAL GROUP}

The full (nonrelativistic) Lagrangian for a Chern-Simons theory based on the Lie group $\mathcal{G}$ is given by

$$
\mathcal{L}=\sum_{m l}\left[i \bar{\Phi}_{l}^{m}\left[\partial_{0}+\vec{a}_{0} \cdot \rho_{m}(\vec{g})+I l c_{0}\right] \Phi_{l}^{m}-\left|\left[\nabla_{i}+\vec{a}_{i} \cdot \rho_{m}(\vec{g})+I l c_{i}\right] \Phi_{l}^{m}\right|^{2}-V\left(\left|\Phi_{l}^{m}\right|\right)\right]+\frac{1}{\pi} \epsilon^{\mu \nu \lambda} A_{\mu} \partial_{\nu} c_{\lambda}+k L_{\mathrm{CS}}(\vec{a}),
$$

where $\vec{g}$ are the generators of the Lie algebra of $\mathcal{G}$, and $\rho_{m}$ is a representation of the Lie algebra. The first term in the third line of Eq. (2) assumes that we have a superconducting condensate of charge 2 bosons. The general Chern-Simons Lagrangian is given by

$$
L_{\mathrm{CS}}(\vec{g})=\frac{1}{4 \pi} \epsilon^{\mu \nu \lambda} \operatorname{tr}\left[a_{\mu} \partial_{\nu} a_{\lambda}+\frac{2}{3} a_{\mu} a_{\nu} a_{\lambda}\right]
$$

Here, $a_{\mu} \equiv \vec{a}_{\mu} \cdot \rho_{\text {adj }}(\vec{g})$ so that the trace is taken in the adjoint representation.

We assume that a single field $\Phi=\Phi_{l}^{m}$ acquires a constant nonzero expectation value. Ignoring gapped fluctuations in $\Phi$ and working in the gauge where $\vec{a}_{0}=0$ and $c_{0}=0$, we acquire the symmetry-breaking Lagrangian

$$
L=-\left|\left[\vec{a}_{i} \cdot \rho(\vec{g})+l I c_{i}\right] \Phi\right|^{2}-V(\Phi)+\frac{k I_{a d}}{4 \pi} \epsilon^{i 0 j} \vec{a}_{i} \cdot B \cdot \partial_{0} \vec{a}_{j}+\frac{1}{\pi} \epsilon^{\mu \nu i} A_{\mu} \partial_{\nu} c_{i}
$$

Once again, we assume that $l \neq 0$. The $U(1)$ field $c_{i}+\frac{1}{l} \vec{a}_{i} \cdot\langle\rho(\vec{g})\rangle$ is clearly gapped. Integrating out this field leads to the effective-mass matrix

$$
M_{\rho(\vec{g})}=\langle\rho(\vec{g}) \rho(\vec{g})\rangle-\langle\rho(\vec{g})\rangle\langle\rho(\vec{g})\rangle
$$

for the remaining gauge fluctuations. This mass matrix is dependent upon the representation of $\mathcal{G}$ carried by $\Phi_{l}^{m}$. As described above, there is a remaining gauge symmetry if and only if $|u\rangle$ is an eigenstate of $\hat{v} \cdot \rho(\vec{g})$ for some vector $\hat{v}$, in which case the gauge field along $\hat{v}$ remains gapless. Let $\vec{s}$ be the set of generators for which $\rho\left(s_{j}\right)|u\rangle=0$. These generators form a subalgebra of $g$, called the stabilizer subalgebra of $g$ on $|u\rangle$. The gauge field along any such $s_{i}$ remains gapless, as both terms in the mass 
matrix vanish. In addition, let $\vec{h}$ be a vector of generators in the Cartan subalgebra $h \subset g$. Then if the field $\vec{v} \cdot \rho(\vec{h})$ remains gapless, one may always make a gauge transformation so that $\vec{v} \cdot \rho(\vec{h})|u\rangle=\vec{v} \cdot \vec{r}|u\rangle$, where $\vec{r}$ is a weight of the representation $\rho$. We find one gapless generator in the Cartan subalgebra that is not in the stabilizer subalgebra, corresponding to the generator $\vec{r} \cdot \rho(\vec{h})$. This is precisely the $\mathrm{U}(1)$ charge mode of the resulting theory.

\section{Example: $G_{2}$ level 1}

As an example, we consider the case of a superconductor with anyons governed by $G_{2}$ at level 1 . The group $G_{2}$ is $14-$ dimensional, with a two-dimensional Cartan subalgebra. This is a Fibonacci superconductor built on a bosonic quantum Hall state. There are no fermions in the superconducting state; the only nontrivial particle is a Fibonacci anyon, which carries the seven-dimensional fundamental representation of $G_{2}$. There are 14 gauge fields in the effective action (2). When the field in $G_{2}$ level 1 Chern-Simons theory associated with the composite of a Fibonacci anyon and flux $l=1$ acquires an expectation value, then the remaining gapless gauge fields result in the (221) bosonic quantum Hall state. To see this, we consider a basis for the fundamental representation in which the weights are given by $(0,0),( \pm 1,0),( \pm 1, \mp 1)$, and $(0, \pm 1)$. The stabilizer subgroup associated with any of the nonzero weights is isomorphic to $\mathrm{SU}(2)$, while that associated with the weight $(0,0)$ is isomorphic to $\mathrm{SU}(3)$. Because both $\mathrm{SU}(3)_{1}$ and $\mathrm{SU}(2)_{1}$ are Abelian, we may proceed by considering only the Cartan subalgebra, leading to an equivalent $U(1) \times U(1)$ theory for the broken phase. In the basis we chose, the Killing form is given by $\left({ }_{-1}^{2} \quad-\frac{1}{2}\right)$, which is equivalent, after an $\operatorname{SL}(2, \mathbb{Z})$ basis change, to the $K$-matrix $\left(\begin{array}{ll}2 & 1 \\ 1 & 2\end{array}\right)$. Under this basis change, the weight vector $\vec{r}=(1,-1)$ is transformed to the standard charge vector for the $(2,2,1)$ state. (Other choices of weight vector [except $(0,0)$ ] all lead to the same state via a $\mathbb{D}_{3}$ symmetry of the $K$ matrix. The weight $r=(0,0)$ leads to a $\mathbb{Z}_{3}$ state at filling $v=0$.) In other words, we obtain the inverse of the construction obtained in Ref. [1] for the bosonic superconducting Fibonacci state.

[1] R. S. K. Mong, D. J. Clarke, J. Alicea, N. H. Lindner, P. Fendley, C. Nayak, Y. Oreg, A. Stern, E. Berg, K. Shtengel et al., Phys. Rev. X 4, 011036 (2014). 